\title{
On the Higgs-like boson in the minimal supersymmetric 3-3-1 model
}

\author{
J. G. Ferreira Jr ${ }^{1}$, C. A. de S. Pires ${ }^{1, a}$, P. S. Rodrigues da Silva ${ }^{1}$, Clarissa Siqueira ${ }^{1,2}$ \\ ${ }^{1}$ Departamento de Física, Universidade Federal da Paraíba, Caixa Postal 5008, João Pessoa, PB 58051-970, Brazil \\ ${ }^{2}$ Max-Planck-Institut für Kernphysik, Saupfercheckweg 1, 69117 Heidelberg, Germany
}

Received: 17 November 2017 / Accepted: 3 March 2018 / Published online: 16 March 2018

(C) The Author(s) 2018

\begin{abstract}
It is imperative that any proposal of new physics beyond the standard model possesses a Higgs-like boson with $125 \mathrm{GeV}$ of mass and couplings with the standard particles that recover the branching ratios and signal strengths as measured by CMS and ATLAS. We address this issue within the supersymmetric version of the minimal 3-3-1 model. For this we develop the Higgs potential with focus on the lightest Higgs provided by the model. Our proposal is to verify if it recovers the properties of the Standard Model Higgs. With respect to its mass, we calculate it up to one loop level by taking into account all contributions provided by the model. In regard to its couplings, we restrict our investigation to couplings of the Higgs-like boson with the standard particles, only. We then calculate the dominant branching ratios and the respective signal strengths and confront our results with the recent measurements of CMS and ATLAS. As distinctive aspects, we remark that our Higgs-like boson intermediates flavor changing neutral processes and has as signature the decay $t \rightarrow h+c$. We calculate its branching ratio and compare it with current bounds. We also show that the Higgs potential of the model is stable for the region of parameter space employed in our calculations.
\end{abstract}

\section{Introduction}

The discovery of a scalar state by the ATLAS and CMS at CERN $[1,2]$ compatible with the standard model Higgs boson was a milestone in particle physics since it was the missing part of the theory. Nowadays we not only know the Higgs mass with precision, $M_{h}=125.09 \pm 0.21$ (stat.) \pm 0.11 (syst.) $\mathrm{GeV}$, but also know that its signal strengths are found consistent with the standard model (SM) predictions [3]. However, the present experimental uncertainties allow

\footnotetext{
a e-mail: cpires@ fisica.ufpb.br
}

us to interpret such observed state as belonging to a more complex theory that encompasses the standard one.

Supersymmetry is the most popular proposal of new physics and all supersymmetric models predict at least one light Higgs-like boson. For example, the minimal supersymmetric standard model (MSSM) provides a tree level contribution to the mass of the Higgs-like boson that does not surpass the neutral gauge boson $Z^{0}$ mass. Consequently the amount of radiative corrections required to complete 125 $\mathrm{GeV}$ demands stops with mass in a region of values that threaten the naturalness principle [4]. In view of this it turns out imperative to examine the Higgs sector of any supersymmetric phenomenological model with the aim of investigating the lightest Higgs concerning its mass and couplings with the standard particles.

In this work we develop the scalar sector of the supersymmetric version of the minimal $S U(3)_{C} \times S U(3)_{L} \times U(1)_{N}$ (331) model. The motivations to study 331 models are various as, for example, explanation of family replication [5,6], electric charge quantization [7-9], strong CP-problem [1012], incorporation of inflation [13] and so forth. As we show in this paper, its SUSY versions, besides solving the hierarchy problem, provides a tree level contribution to the lightest Higgs boson that may surpass $100 \mathrm{GeV}$. This is nice in what concerns the naturalness principle since now $125 \mathrm{GeV}$ Higgs is compatible with stop with mass below the $\mathrm{TeV}$ scale.

The minimal supersymmetric 331 model was firstly developed in [14]. For subsequent works, see [15-17]. As the Higgs sector of the minimal 331 model $[5,6]$ requires at least three Higgs triplets to generate correctly the masses of all massive particles and be phenomenologically viable [18-20], then its supersymmetric version, what we call the minimal SUSY331 model, must involve six Higgs triplets as required to cancel anomalies. On assuming that the two triplets $\chi$ and $\chi^{\prime}$ decouple from the other four $\eta, \rho, \eta^{\prime}$ and $\rho^{\prime}$, and that $\eta$ and $\rho$ are inert, then after spontaneous breaking of the 331 symmetry to the standard one, we obtain the 
potential for two Higgs doublets modified by cubic invariant terms. On developing such potential, we obtain the following approximate expression for the lightest scalar, the Higgs-like boson candidate, of the model

$M_{h}^{2} \leq 4 M_{Z}^{2} \sin ^{2}\left(\theta_{w}\right)\left(1+\cos ^{2}(\beta)\right)^{2}$,

where $M_{Z}$ is the mass of the standard neutral gauge boson, $\theta_{w}$ is the electroweak mixing angle and the angle $\beta$ is such that $\tan \beta=\frac{v_{\eta^{\prime}}}{v_{\rho^{\prime}}}$. According to this expression the tree level contribution to the lightest Higgs may attain $174 \mathrm{GeV}$ for $\cos (\beta)=1$. This is so because the symmetries of the model allow the superpotential to have cubic invariant terms formed with the scalar triplets of the model. This acts like in the NMSSM [14]. In view of this, it is compulsory to review the Higgs sector of this model in the light of the recent discoveries of the LHC, and verifying how realistic the expression above is and define precisely the profile of the lightest Higgs in the minimal SUSY331 model.

Our contribution to this discussion restricts to obtain the mass matrix that contains the neutral Higgs, diagonalize it in the most general way and obtain the eigenvalue and the eigenvector that corresponds to the lightest Higgs. We investigate the behavior of its mass by taking into account one loopcorrections. We also obtain the branching ratios for the dominant processes and calculate the respective signal strengths. It is notorious that our Higgs-like boson candidate intermediates flavor changing processes. We discuss such processes but focusing on the dominant ones that are $t \rightarrow h+c$ and $t \rightarrow h+u$. Finally, we guarantee that the region of parameter space employed in our calculations leads to a stable potential.

The paper is divided in the following way: In Sect. 2 we present the main ingredients of the model. Next, in Sect. 3, we develop the Higgs sector with focus on the lightest Higgs where we calculate its mass up to one-loop level. In Sect. 4 we calculate its branching ratios and the respective signal strengths. In Sect. 5 we calculate the flavor changing processes. In Sect. 6 we address the stability of the potential and, finally, we conclude in Sect. 7.

\section{The main ingredients of the model}

In this section we present the core of the minimal SUSY331 model relevant for what follows. In regard to the leptonic sector, the superfields of each generation are arranged in triplet of superfields according to the following transformation by the 3-3-1 symmetry

$\hat{L}_{a}=\left(\begin{array}{c}\hat{v}_{a} \\ \hat{e}_{a} \\ \hat{e}_{a}^{c}\end{array}\right)_{L} \sim(1,3,0)$, where $a=1,2,3$ represents the family index for the usual three generations of leptons.

In the Hadronic sector, the superfields of the third generation comes in the triplet representation and the superfields of the other two come in anti-triplet representations of $S U(3)_{L}$, as a requirement for anomaly cancellation. They are given by

$$
\begin{aligned}
& \hat{Q}_{\alpha_{L}}=\left(\begin{array}{c}
\hat{d}_{\alpha} \\
\hat{u}_{\alpha} \\
\hat{j}_{\alpha}^{\prime}
\end{array}\right)_{L} \sim\left(3,3^{*},-\frac{1}{3}\right), \hat{u}_{\alpha_{L}}^{c} \sim\left(3^{*}, 1,-\frac{2}{3}\right), \\
& \hat{d}_{\alpha_{L}}^{c} \sim\left(3^{*}, 1,1 / 3\right), \hat{j}_{\alpha_{L}}^{c} \sim\left(3^{*}, 1, \frac{4}{3}\right), \\
& \hat{Q}_{3 L}=\left(\begin{array}{c}
\hat{u}_{3} \\
\hat{d}_{3} \\
\hat{j}_{3}^{\prime}
\end{array}\right)_{L} \sim\left(3,3, \frac{2}{3}\right), \hat{u}_{3_{L}}^{c} \sim\left(3^{*}, 1,-\frac{2}{3}\right), \\
& \hat{d}_{3_{L}}^{c} \sim\left(3^{*}, 1,1 / 3\right), \hat{j}_{3 L}^{c} \sim\left(3^{*}, 1,-\frac{5}{3}\right),
\end{aligned}
$$

where $\alpha=1,2$.

The scalar sector of the 3-3-1 model, responsible for the spontaneously broken gauge symmetry, is composed by three scalar triplets. In the supersymmetric version, anomaly cancellation requires we double these fields. Thus, the scalar sector of the minimal SUSY331 is composed by the following superfields

$\hat{\eta}=\left(\begin{array}{c}\hat{\eta} \\ \hat{\eta}_{1}^{-} \\ \hat{\eta}_{2}^{+}\end{array}\right), \hat{\chi}=\left(\begin{array}{c}\hat{\chi}^{-} \\ \hat{\chi}^{--} \\ \hat{\chi}\end{array}\right), \hat{\rho}=\left(\begin{array}{c}\hat{\rho}^{+} \\ \hat{\rho} \\ \hat{\rho}^{++}\end{array}\right)$,

where $\hat{\eta} \sim(1,3,0), \hat{\chi} \sim(1,3,-1), \quad \hat{\rho} \sim(1,3,1)$, and

$\hat{\eta}^{\prime}=\left(\begin{array}{c}\hat{\eta}^{\prime} \\ \hat{\eta}_{1}^{\prime+} \\ \hat{\eta}_{2}^{\prime-}\end{array}\right), \hat{\chi}^{\prime}=\left(\begin{array}{c}\hat{\chi}^{\prime+} \\ \hat{\chi}^{\prime++} \\ \hat{\chi}^{\prime}\end{array}\right), \hat{\rho}^{\prime}=\left(\begin{array}{c}\hat{\rho}^{\prime-} \\ \hat{\rho}^{\prime} \\ \hat{\rho}^{\prime--}\end{array}\right)$,

where $\hat{\eta}^{\prime} \sim\left(1,3^{*}, 0\right), \hat{\chi}^{\prime} \sim\left(1,3^{*}, 1\right), \hat{\rho}^{\prime} \sim\left(1,3^{*},-1\right)$.

We assume that the neutral scalars $\eta, \eta^{\prime}, \rho, \rho^{\prime}, \chi$ and $\chi^{\prime}$ develop nonzero VEV according to

$$
\begin{aligned}
\langle\eta\rangle & =\frac{v_{\eta}}{\sqrt{2}},\left\langle\eta^{\prime}\right\rangle=\frac{v_{\eta^{\prime}}}{\sqrt{2}},\langle\rho\rangle=\frac{v_{\rho}}{\sqrt{2}}, \\
\left\langle\rho^{\prime}\right\rangle & =\frac{v_{\rho^{\prime}}}{\sqrt{2}},\langle\chi\rangle=\frac{v_{\chi}}{\sqrt{2}},\left\langle\chi^{\prime}\right\rangle=\frac{v_{\chi^{\prime}}}{\sqrt{2}} .
\end{aligned}
$$

These VEVs lead to the following gauge symmetry breaking pattern

$$
\begin{aligned}
S U(3)_{C} & \otimes S U(3)_{L} \otimes U(1)_{X} \stackrel{v_{\chi}, v_{\chi^{\prime}}}{=} S U(3)_{C} \otimes S U(2)_{L} \\
& \otimes U(1)_{Y} \stackrel{v_{\eta}, v_{\eta^{\prime}}, v_{\rho}, v_{\rho^{\prime}}}{\Longrightarrow} S U(3)_{C} \otimes U(1)_{Q E D} .
\end{aligned}
$$

With the breaking of the gauge symmetry by this set of VEVs all the massive particles, including the supersymmetric ones, receive mass. What matters for us here are the scalar and gauge boson masses. Concerning the gauge bosons, they are 
composed by the standard gauge boson, $\gamma, Z^{0}$ and $W^{ \pm}$, one new neutral massive gauge bosons $Z^{\prime}$, two doubly charged gauge bosons $U^{ \pm \pm}$, and two simply charged gauge bosons $V^{ \pm}$. The charged gauge bosons gain the following mass expressions

$$
\begin{aligned}
M_{W^{ \pm}} & =\frac{g^{2}}{4}\left(v_{\eta}^{2}+v_{\eta^{\prime}}^{2}+v_{\rho}^{2}+v_{\rho^{\prime}}^{2}\right) \\
M_{V^{ \pm}} & =\frac{g^{2}}{4}\left(v_{\eta}^{2}+v_{\eta^{\prime}}^{2}+v_{\chi}^{2}+v_{\chi^{\prime}}^{2}\right) \\
M_{U^{ \pm \pm}} & =\frac{g^{2}}{4}\left(v_{\rho}^{2}+v_{\rho^{\prime}}^{2}+v_{\chi}^{2}+v_{\chi^{\prime}}^{2}\right),
\end{aligned}
$$

while the neutral gauge bosons have masses

$$
\begin{aligned}
& M_{Z}=g^{2} \frac{\left(1+4 t^{2}\right)}{\left(4+12 t^{2}\right)}\left(v_{\eta}^{2}+v_{\eta^{\prime}}^{2}+v_{\rho}^{2}+v_{\rho^{\prime}}^{2}\right) \\
& M_{Z^{\prime}}=\frac{1}{3} g^{2}\left(3 t^{2}+1\right)\left(v_{\chi}^{2}+v_{\chi^{\prime}}^{2}\right),
\end{aligned}
$$

where $t=g_{N} / g, v_{\rho}^{2}+v_{\rho^{\prime}}^{2}+v_{\eta}^{2}+v_{\eta^{\prime}}^{2}=v_{e w}^{2}$ and $v_{\chi}^{2}+v_{\chi^{\prime}}^{2} \equiv$ $v_{331}^{2}$ with $v_{331}$ lying in the $\mathrm{TeV}$ scale.

After all this, we are ready to build up the superpotential of the model. The superpotential that respects the gauge symmetry and R-parity is composed by the following terms

$$
\begin{aligned}
W= & \mu_{\eta} \hat{\eta} \hat{\eta}^{\prime}+\mu_{\rho} \hat{\rho} \hat{\rho}^{\prime}+\mu_{\chi} \hat{\chi} \hat{\chi}^{\prime} \\
& +f_{1} \hat{\rho} \hat{\chi} \hat{\eta}+f_{1}^{\prime} \hat{\rho}^{\prime} \hat{\chi}^{\prime} \hat{\eta}^{\prime}+\sum_{i}\left(\frac{\kappa_{1 i j}}{\Lambda} \hat{L}_{j} \hat{\rho}^{\prime} \hat{\chi}^{\prime} \hat{L}_{i}\right) \\
& +\sum_{i, \alpha}\left(\kappa_{2 i \alpha} \hat{Q}_{\alpha} \hat{\eta} \hat{d}_{i}^{c}+\kappa_{3 i \alpha} \hat{Q}_{\alpha} \hat{\rho} \hat{u}_{i}^{c}\right) \\
& +\sum_{\alpha, \beta}\left(\kappa_{4 \alpha \beta} \hat{Q}_{\alpha} \hat{\chi} \hat{j}_{\beta}^{c}\right) \\
& +\sum_{i}\left(\kappa_{5 i} \hat{Q}_{3} \hat{\eta}^{\prime} \hat{u}_{i}^{c}+\kappa_{6 i} \hat{Q}_{3} \hat{\rho}^{\prime} \hat{d}_{i}^{c}\right)+\kappa_{6} \hat{Q}_{3} \hat{\chi}^{\prime} \hat{j}_{3}^{c},
\end{aligned}
$$

where $\alpha, \beta=1,2$ and $i=1,2,3$. We could be economical and resort to a $Z_{3}$ symmetry so as to avoid the bilinear terms in the superpotential above. However, in order to be general enough, we do not follow this path here.

We call the attention to the fact that the last term in the first line of the superpotential is an effective 5-D operator. It will generate the masses of the charged leptons. This point has been well developed in many previous papers, but it is appropriate to recall it here. The highest energy scale where the model is found to be perturbatively reliable is about $\Lambda=$ $4-5 \mathrm{TeV}$. Hence, effective operators may be required to generate corrections to the mass of some charged fermions. We make use of this here with the aim of avoiding the addition of scalars sextets to the model.

Up to this point the masses of the standard particles are equal to the masses of their superpartners. As usual, in phenomenological supersymmetric models SUSY must be broken so as to provide a reasonable shift between ordinary par- ticles and their supersymmetric partners. In this work we assume that SUSY is broken explicitly through the following set of soft breaking terms that are invariant under the symmetries assumed here

$$
\begin{aligned}
\mathcal{L}_{\text {soft }}= & -\frac{1}{2}\left(m_{\lambda_{c}} \sum_{a}\left(\lambda_{c}^{a} \lambda_{c}^{a}\right)+m_{\lambda} \sum_{a}\left(\lambda^{a} \lambda^{a}\right)+m_{\lambda}^{\prime} \lambda \lambda+h . c .\right) \\
& -m_{L}^{2} \tilde{L}^{\dagger} \tilde{L}-m_{Q_{\alpha}}^{2} \tilde{Q}_{\alpha}^{\dagger} \tilde{Q}_{\alpha}+\sum_{i}\left(\tilde{u}_{i}^{\dagger} m_{u_{i}}^{2} \tilde{u}_{i}-\tilde{d}_{i}^{\dagger} m_{d_{i}}^{2} \tilde{d}_{i}\right) \\
& -m_{J}^{2} \tilde{j}_{3}^{\dagger} \tilde{j}_{3}-\sum_{\beta} \tilde{j}_{\beta} m_{j \beta}^{2} \tilde{j}_{\beta}-\sum_{\alpha} m_{Q_{3}}^{2} \tilde{Q}_{3}^{\dagger} \tilde{Q}_{3}-m_{\eta}^{2} \eta^{\dagger} \eta \\
& -m_{\rho}^{2} \rho^{\dagger} \rho-m_{\chi}^{2} \chi^{\dagger} \chi-m_{\eta}^{\prime 2} \eta^{\prime \dagger} \eta^{\prime}-m_{\rho}^{\prime 2} \rho^{\prime \dagger} \rho^{\prime} \\
& -m_{\chi}^{\prime 2} \chi^{\prime \dagger} \chi^{\prime \dagger}+k_{1} \rho \chi \eta+k_{2} \rho^{\prime} \chi^{\prime} \eta^{\prime}+b_{\rho} \rho^{\prime} \rho+b_{\eta} \eta^{\prime} \eta+b_{\chi} \chi^{\prime} \chi \\
& +\sum_{\alpha} \tilde{Q}_{\alpha}\left[\sum_{i}\left(\omega_{1 \alpha i} \eta \tilde{d}_{i}^{c}+\omega_{2 \alpha i} \rho \tilde{u}_{i}^{c}\right)+\sum_{\beta} \omega_{3 \alpha \beta} \chi \tilde{j}_{\beta}^{c}+h . c .\right] \\
& +\sum_{i} \tilde{Q}_{3}\left(\xi_{1 i} \eta^{\prime} \tilde{u}_{i}^{c}+\xi_{2 i} \rho^{\prime} \tilde{d}_{i}^{c}+\xi_{3} \chi^{\prime} \tilde{j}_{3}^{c}\right) .
\end{aligned}
$$

where $\alpha, \beta=1,2$ and $i=1,2,3 . \lambda_{c}^{a}$ are the gluinos, $\lambda^{a}$ are gauginos associated to $S U(3)_{L}$ (in both cases $a=1, \ldots, 8$ is the gauge group index) and $\lambda$ is the gaugino associated to $U(1)_{N}$. The scalar supersymmetric partners of fermion fields, $f$, are denoted by $\tilde{f}$, while the remaining fields are self-evident. For simplicity, we will take $m_{L}^{2}=m_{Q_{\alpha}}^{2}=$ $m_{Q_{3}}^{2}=m_{S U S Y_{L}}^{2}, m_{u_{i}}^{2}=m_{d_{i}}^{2}=m_{j \beta}^{2}=m_{S U S Y_{R}}^{2}$ and $m_{J}^{2}=10 \times m_{S U S Y_{R}}^{2}$.

As it is usual in SUSY models, which involves a large number of free parameters, simplifications are necessary in order to easily get a better view of the big picture. As simplification we use the following parametrization

$$
\begin{aligned}
b_{\rho, \eta, \chi} & =B_{0} \times \mu_{\rho, \eta, \chi} ; \quad \omega, \xi=A_{0} \times \kappa ; M_{s}=\left(m_{\tilde{t}_{1}} m_{\tilde{t}_{2}}\right)^{1 / 2}, \\
v_{331} & =\sqrt{v_{\chi}^{2}+v_{\chi^{\prime}}^{2}} ; \tan \beta=\frac{\sqrt{v_{\eta}^{2}+v_{\eta^{\prime}}^{2}}}{\sqrt{v_{\rho}^{2}+v_{\rho^{\prime}}^{2}}}, \tan \beta_{\eta}=\frac{v_{\eta^{\prime}}}{v_{\eta}} ; \\
\tan \beta_{\rho} & =\frac{v_{\rho^{\prime}}}{v_{\rho}} ; \\
\tan \beta_{\chi} & =\frac{v_{\chi^{\prime}}}{v_{\chi}}, \\
X_{t} & =\xi_{13}+\mu_{\eta} \cot \beta_{\eta}+f_{1}^{\prime} v_{331} \cot \beta \frac{\sin \beta_{\rho} \sin \beta_{\chi}}{\sin \beta_{\eta}}, \quad \text { (11) }
\end{aligned}
$$

where $\kappa$ 's are the corresponding Yukawa couplings, $M_{S}$ is the SUSY scale, $m_{\tilde{t}}$ is the mass of the stop and $X_{t}$ is the equivalent of the soft trilinear coupling of the stops. We also assume that all soft left and right masses, $m_{S U S Y_{L}}$ and $m_{S U S Y_{R}}$, are equals. With all this we are ready to further explore the Higgs physics. 


\section{Higgs physics in the minimal SUSY331 model}

In supersymmetric models the Higgs potential receives contributions from three different sources, F-term, D-term and the soft SUSY breaking terms, that adds up to form the scalar potential $V=V_{F}+V_{D}+V_{\text {soft }}$. These contributions are, respectively

$$
\begin{aligned}
& V_{F}=\mu_{\eta}^{2}|\eta|^{2}+\mu_{\eta}^{2}\left|\eta^{\prime}\right|^{2}+\mu_{\rho}^{2}|\rho|^{2}+\mu_{\rho}^{2}\left|\rho^{\prime}\right|^{2} \\
& +\mu_{\chi}^{2}|\chi|^{2}+\mu_{\chi}^{2}\left|\chi^{\prime}\right|^{2} \\
& +f_{1}^{2}\left(|\eta|^{2}|\rho|^{2}-|\eta \cdot \rho|^{2}\right) \\
& +f_{1}^{2}\left(|\eta|^{2}|\chi|^{2}-|\eta \cdot \chi|^{2}\right) \\
& +f_{1}^{2}\left(|\chi|^{2}|\rho|^{2}-|\chi \cdot \rho|^{2}\right) \\
& +f_{1}^{\prime 2}\left(\left|\eta^{\prime}\right|^{2}\left|\rho^{\prime}\right|^{2}-\left|\eta^{\prime} \cdot \rho^{\prime}\right|^{2}\right) \\
& +f_{1}^{\prime 2}\left(\left|\eta^{\prime}\right|^{2}\left|\chi^{\prime}\right|^{2}-\left|\eta^{\prime} \cdot \chi^{\prime}\right|^{2}\right) \\
& +f_{1}^{\prime 2}\left(\left|\chi^{\prime}\right|^{2}\left|\rho^{\prime}\right|^{2}-\left|\chi^{\prime} \cdot \rho^{\prime}\right|^{2}\right) \\
& -f_{1} \epsilon_{i j k}\left(\mu_{\eta} \eta_{i}^{\prime \dagger} \rho_{j} \chi_{k}+\mu_{\rho} \eta_{i} \rho_{j}^{\prime \dagger} \chi_{k}\right. \\
& +\mu_{\chi} \eta_{i} \rho_{j} \chi_{k}^{\prime \dagger}+\text { h.c.) } \\
& -f_{1}^{\prime} \epsilon_{i j k}\left(\mu_{\eta} \eta_{i} \rho_{j}^{\prime \dagger} \chi_{k}^{\prime \dagger}\right. \\
& \left.+\mu_{\rho} \eta_{i}^{\prime \dagger} \rho_{j} \chi_{k}^{\prime \dagger}+\mu_{\chi} \eta_{i}^{\prime \dagger} \rho_{j}^{\prime \dagger} \chi_{k}+\text { h.c. }\right) \text {, } \\
& V_{D}=\frac{g^{2}}{2} \sum_{A}\left(\rho^{\dagger} t_{A} \rho-\rho^{\prime \dagger} t_{A}^{*} \rho^{\prime}+\eta^{\dagger} t_{A} \eta-\eta^{\prime \dagger} t_{A}^{*} \eta^{\prime}\right. \\
& \left.+\chi^{\dagger} t_{A} \chi-\chi^{\prime \dagger} t_{A}^{*} \chi^{\prime}\right)^{2} \\
& +\frac{g_{N}^{2}}{2}\left(\rho^{\dagger} \rho-\rho^{\prime \dagger} \rho^{\prime}-\chi^{\dagger} \chi\right. \\
& \left.+\chi^{\prime \dagger} \chi^{\prime}\right)^{2} \text {, } \\
& V_{\text {soft }}=-m_{\eta}^{2} \eta^{\dagger} \eta-m_{\rho}^{2} \rho^{\dagger} \rho-m_{\chi}^{2} \chi^{\dagger} \chi-m_{\eta^{\prime}}^{2} \eta^{\prime \dagger} \eta^{\prime} \\
& -m_{\rho^{\prime}}^{2} \rho^{\prime \dagger} \rho^{\prime}-m_{\chi^{\prime}}^{2} \chi^{\prime \dagger} \chi^{\prime}+b_{\rho} \rho^{\prime} \rho \\
& +b_{\eta} \eta^{\prime} \eta+b_{\chi} \chi^{\prime} \chi+k_{1} \rho \chi \eta+k_{2} \rho^{\prime} \chi^{\prime} \eta^{\prime} \text {, }
\end{aligned}
$$

where $t_{A}$ are the Gell-Mann matrices.

The fields are assumed to be shifted in the usual way

$$
\begin{aligned}
& \rho^{0}, \rho^{0}, \eta^{0}, \eta^{\prime 0} \chi^{0}, \chi^{\prime 0} \\
& \quad \rightarrow \frac{1}{\sqrt{2}}\left(v_{\rho, \rho^{\prime}, \eta, \eta^{\prime} \chi, \chi^{\prime}}+R_{\rho, \rho^{\prime}, \eta, \eta^{\prime} \chi, \chi^{\prime}}+i I_{\rho, \rho^{\prime}, \eta, \eta^{\prime} \chi, \chi^{\prime}}\right),
\end{aligned}
$$

and the set of minimum conditions are given in Appendix A. That set of constraint equations enable us to obtain the mass matrix of the scalars of the model.

In this work we focus exclusively in the lightest neutral scalar which is expected to recover the properties of the Standard Model Higgs in what concerns its mass, branching ratios and signal strengths. For this we first obtain the $6 \times 6$ mass matrix, $M_{R_{H}}^{2}$, associated to the CP-even scalars $R_{\eta}, R_{\eta^{\prime}}, R_{\rho}, R_{\rho^{\prime}}, R_{\chi}$ and $R_{\chi^{\prime}}$. It is a very complex, and not illuminating, mass matrix, then we do not show it here. Next, we diagonalize it by a rotating mixing matrix $U_{R}$ such that the diagonal mass matrix $M_{H_{D}}^{2}$ relates to the $M_{R_{H}}^{2}$ through the relation $M_{H_{D}}^{2}=U_{R}^{\dagger} M_{R_{H}}^{2} U_{R}$. The physical eigenstates relates to the symmetrical ones by $H=U_{R} R$ where $H=\left(h_{1}, h_{2}, h_{3}, h_{4}, h_{5}, h_{6}\right)^{T}$ and $R=\left(R_{\eta}, R_{\eta^{\prime}}, R_{\rho}, R_{\rho^{\prime}}, R_{\chi}, R_{\chi^{\prime}}\right)^{T}$. The lightest eigenstate, let us call it $h_{i}$, must be identified as the Higgs-like boson. This means that it must have a mass of $125 \mathrm{GeV}$ and its eigenvector, $h_{i}=\left(U_{R}\right)_{i j} R_{j}$, must recover the standard Higgs couplings. In practical terms, to know the eigenvector $h_{i}$ means to obtain the set of entries $\left(U_{R}\right)_{i j}$. Such entries, when combined with the adequate couplings, must recover the existing branching ratios and signal strengths of the Higgs. From now on we refer to the eigenvector associated to the Higgs-like boson as $h$.

Due to the large number of parameters involved in the diagonalization of $M_{R_{H}}^{2}$, an analytical approach is unviable. In view of this our approach will be completely numerical. In what follows we make use of powerful tools like the packages Sarah 4.10.2 [21-23], Spheno 4.0.2 [24,25] and SSP 1.2.3 [26] ( We include in Appendix B and C the implemented model in Sarah and the Spheno input, respectively). Throughout this work we employ the following routine: we implement the model in the Sarah 10.2 and export it to the Spheno 4.0.2, where we make all the numerical calculations. The SSP helps in making the scan of the parameters. Hereafter all numerical computations done in this paper follow this routine, including the diagonalization of this mass matrix.

\subsection{Tree level contribution}

Differently from the MSSM, where the tree level contribution to the Higgs mass does not surpass $91 \mathrm{GeV}$, in the minimal SUSY331 model things are a little different. The expression in Eq. (1) predicts that the lightest scalar, the Higgs-like boson, may attain a mass of $125 \mathrm{GeV}$ already at tree level. It is important to note that the approximations assumed in Eq. (1) do not take into account the stability of the potential. Thus, it becomes mandatory, and challenging, to clarify if the general case, which involves the diagonalization of a $6 \times 6$ mass matrix, agrees with the prediction in Eq. (1).

The results displayed in Fig. 1 is for the range of parameters

$$
\begin{aligned}
& -0.03 \leq f_{1} \leq 0.2,0.5 \leq f_{1}^{\prime} \leq 0.6, \\
& -2000 \mathrm{GeV} \leq k_{1} \leq 2000 \mathrm{GeV},-1000 \mathrm{GeV} \\
& \leq k_{2} \leq 500 \mathrm{GeV},-800 \mathrm{GeV} \leq \mu_{\eta} \leq-500 \mathrm{GeV}, \\
& -1800 \mathrm{GeV} \leq \mu_{\rho} \leq-1600 \mathrm{GeV},-1800 \mathrm{GeV} \leq \mu_{\chi} \\
& \leq-1400 \mathrm{GeV}, 2000 \mathrm{GeV} \leq v_{331} \leq 4000 \mathrm{GeV},
\end{aligned}
$$




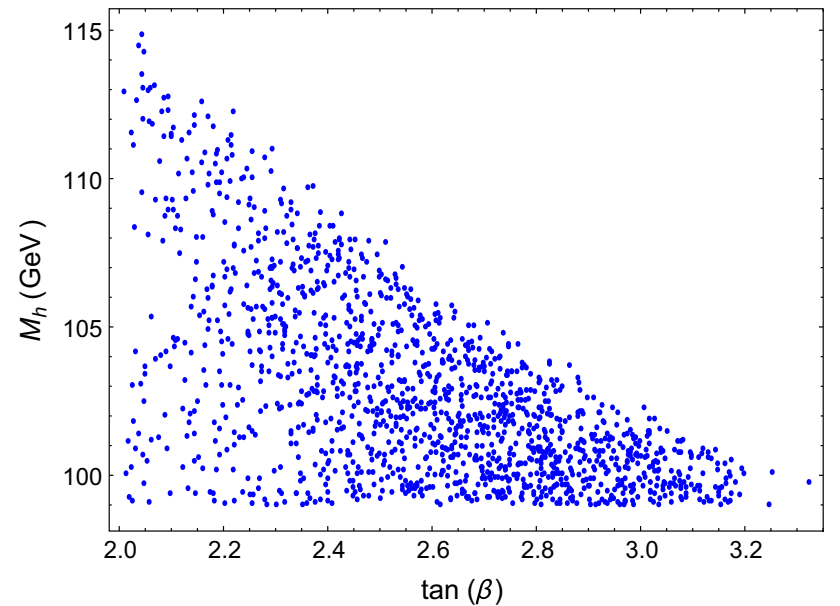

Fig. 1 Tree level contribution to the Higgs-like boson mass as function of $\tan (\beta)$

$$
\begin{aligned}
& 2 \leq \tan \beta \leq 4,8 \leq\left(\tan \beta_{\eta}, \tan \beta_{\rho}\right) \leq 10 \\
& 0.95 \leq \tan \beta_{\chi} \leq 1.20 \\
& v_{\rho}^{2}+v_{\eta}^{2}+v_{\eta^{\prime}}^{2}+v_{\rho^{\prime}}^{2}=(246 \mathrm{GeV})^{2}, A_{0}, B_{0}=1000 \mathrm{GeV} .
\end{aligned}
$$

Observe that the tree level contribution may attain 115 $\mathrm{GeV}$ for $\tan (\beta)=2$ which is in a good agreement with the predictions of the Eq. (1). In other words, our results confirm that Eq. (1) is a valid approximation for the tree level contribution to the Higgs-like boson mass in the minimal SUSY331 model. Thus, the robustness of the Higgs-like boson mass at tree level is dictated by $\tan (\beta)$ such that the smaller $\tan (\beta)$, the larger tree level Higgs-like boson mass.

Nevertheless, we know that loop correction to the Higgs mass are considerable, enforcing us to conclude that $\tan (\beta) \geq$ 2 and that the model may support a stop with mass below the $\mathrm{TeV}$ scale as required by naturalness principle.

For sake of completeness, we obtain the eigenvector associated to the eigenvalue $125 \mathrm{GeV}$ for the following set of values of the parameters

$$
\begin{aligned}
\tan (\beta) & =2.7, \tan \left(\beta_{\eta}\right)=10, \tan \left(\beta_{\rho}\right)=10, \\
\tan \left(\beta_{\chi}\right) & =1.2, v_{\eta}=22.3 \mathrm{GeV}, v_{\eta^{\prime}}=223.2 \mathrm{GeV}, \\
v_{\rho} & =8.1 \mathrm{GeV}, v_{\rho^{\prime}}=81 \mathrm{GeV}, \\
v_{\chi} & =1763.6 \mathrm{GeV}, v_{\chi^{\prime}}=2116.4 \mathrm{GeV}, f_{1}=0.01, \\
f_{1}^{\prime} & =0.6, k_{1}=-500 \mathrm{GeV}, \quad k_{2}=-600 \mathrm{GeV}, \\
b_{\eta} & =-55000 \mathrm{GeV}^{2}, \quad b_{\rho}=-1.7 \times 10^{6} \mathrm{GeV}^{2}, \\
b_{\chi} & =-1.4 \times 10^{6} \mathrm{GeV}^{2}, \mu_{\rho}=-1700 \mathrm{GeV}, \\
\mu_{\eta} & =-550 \mathrm{GeV}, \quad \mu_{\chi}=-1400 \mathrm{GeV} .
\end{aligned}
$$

In this case, the eigenvector corresponding to the $125 \mathrm{GeV}$ Higgs is

$$
\begin{aligned}
h= & -0.93 R_{\eta^{\prime}}-0.34 R_{\rho^{\prime}}-0.09 R_{\eta}-0.03 R_{\rho} \\
& -0.05 R_{\chi^{\prime}}-0.06 R_{\chi} .
\end{aligned}
$$

See that the eigenvector $h$ is composed mainly by $R_{\eta^{\prime}}$ and $R_{\rho^{\prime}}$. For any other choice of the set of values of the parameters, the eigenvector will keep being dominantly a composition of $R_{\eta^{\prime}}$ and $R_{\rho^{\prime}}$.

\subsection{One loop level contribution}

In the MSSM a $125 \mathrm{GeV}$ Higgs demands robust loop corrections which requires stop heavy enough for threatening the naturalness principle. In the minimal SUSY331 model things are a little different once tree level contribution to the Higgs-like boson mass may surpass $100 \mathrm{GeV}$.

Because our model deserves small loop corrections to obtain the wanted $125 \mathrm{GeV}$ Higgs mass, in what follows we calculate numerically, by employing the package Spheno 4.0.2 by performing the classic "one-scale" matching, the Higgs-like boson mass taking into account one loop corrections, only. Our results are displayed in graphics showing the behavior of our Higgs-like boson mass with the parameters $M_{s}, X_{t}$ and $v_{331}$. Our analysis is done within the following set of parameters

$$
\begin{aligned}
f_{1}^{\prime} & =0.6,-0.03 \leq f_{1} \leq 0.2, k_{1}=-500 \mathrm{GeV}, \\
k_{2} & =-600 \mathrm{GeV}, \mu_{\eta}=-550 \mathrm{GeV} \\
\mu_{\rho} & =-1700 \mathrm{GeV}, \mu_{\chi}=-1400 \mathrm{GeV} \\
\tan \beta_{\eta} & =\tan \beta_{\rho}=10, \tan \beta_{\chi}=1.20 \\
1000 \mathrm{GeV} & \leq v_{331} \leq 5000 \mathrm{GeV}, 2 \leq \tan \beta \leq 10 \\
500 \mathrm{GeV} & \leq m_{S U S Y_{L}}, m_{S U S Y_{R}} \leq 2000 \mathrm{GeV} \\
-3000 & \leq X_{t} \leq 3000, \quad A_{0}, B_{0}=1000 \mathrm{GeV}
\end{aligned}
$$

In Fig. 2 we plot $M_{h}$ versus $X_{t}$. As we can see in that plot, small $X_{t}$ is allowed for large value of $v_{331}$, while large $X_{t}$ is allowed by small values of $v_{331}$. Moreover, we highlight that

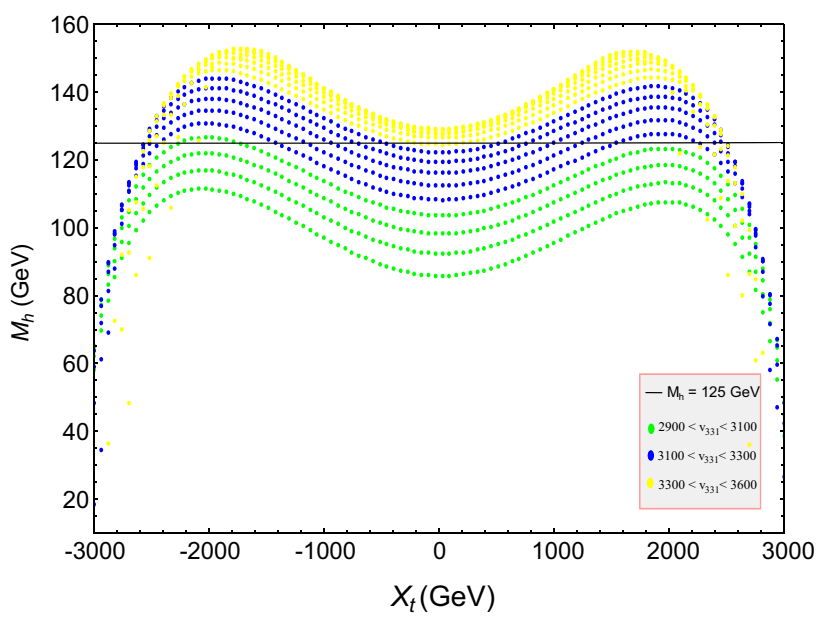

Fig. $2 M_{h}$ vs $X_{t}$ for various values of $v_{331}$. We took $m_{S U S Y_{L}}=$ $m_{S U S Y_{R}}=[500,1000] \mathrm{GeV}$ 


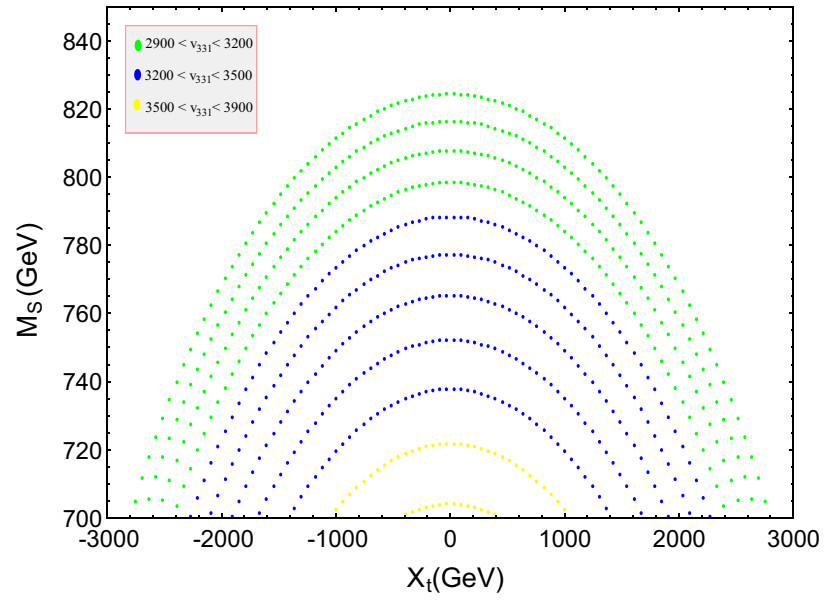

Fig. $3 M_{S}$ versus $X_{t}$ for various values of $v_{331}$

the model provides a $125 \mathrm{GeV}$ Higgs mass even for $X_{t}=0$ and stop mass below $\mathrm{TeV}$ scale.

We also obtained the $2 \times 2$ mass matrix for the stops and diagonalized it with the Spheno package. The result is displayed in Fig. 3 where we show the behavior of $M_{s}$ with $X_{t}$. Note that the larger $v_{331}$, the smaller $M_{s}$. In Fig. 4 (left) we show the behavior of $M_{h}$ with $M_{s}$ for the specific case $X_{t}=0$, and in Fig. 4 (right) we show the behavior of $M_{h}$ with $v_{331}$ for $X_{t}=0$. Perceive that the model provides easily a $125 \mathrm{GeV}$ Higgs boson for $M_{s}$ and $X_{t}$, both, below the TeV scale.

As we can see, the minimal SUSY331 model naturally recovers the Standard Model Higgs boson mass, but we know that it is different from the MSSM one because of the peculiarities of the minimal SUSY331 model. Thus, in order to conclude definitely that our Higgs-like boson recovers all the observed properties of the Standard Model Higgs, we need to calculate its branching ratios and the respective signal strengths and confront the results with the experimental data measured by LHC.

\section{Branching ratios and signal strengths}

In what concerns the Standard Model Higgs, any extension of the SM must possess a scalar with $125 \mathrm{GeV}$ of mass and couplings with the Standard Model particles that fit the measured branching ratios and signal strengths [27]. This is the reason why we wonder if the Higgs-like boson discussed here recovers the Higgs branching ratios and signal strengths as observed by LHC.

As we know, the Higgs prefers to decay into pairs of $b \bar{b}$, $W W^{*}, \tau \bar{\tau}, Z Z^{*}$ and $\gamma \gamma$. We restrict our investigation for these channels. Our results are shown in Fig. 5. Perceive that the predictions are in perfect agreement with the values measured by ATLAS [28] and CMS [29]. In this point of the work, we are secure in affirming that the minimal SUSY331 model is privileged in what concerns Higgs physics since it possesses a Higgs-like boson with mass and couplings with the standard particles that recovers its observed properties and respecting the naturalness principle.

\section{Flavor changing neutral current as signature of our Higgs-like boson}

As we saw above, the lightest Higgs of the minimal SUSY331 model fulfills the conditions to be a SM-like Higgs. However, it is important to recall that our Higgs-like bosom is different from the SM-like one in many aspects with the major one being that it intermediates flavor changing processes involv-
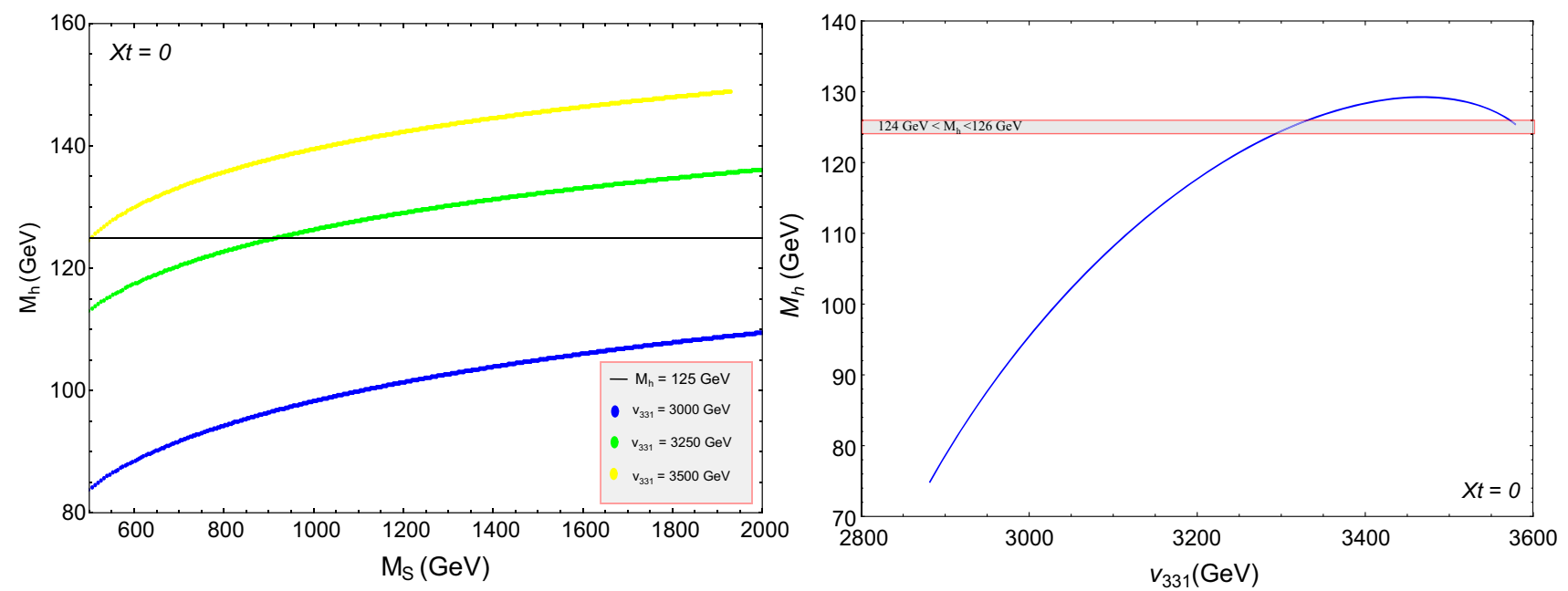

Fig. 4 Left: $M_{h}$ versus $M_{s}$ for $X_{t}=0$ for three different values of $v_{331}$. Right: $M_{h}$ versus $v_{331}$ for $X_{t}=0$ 


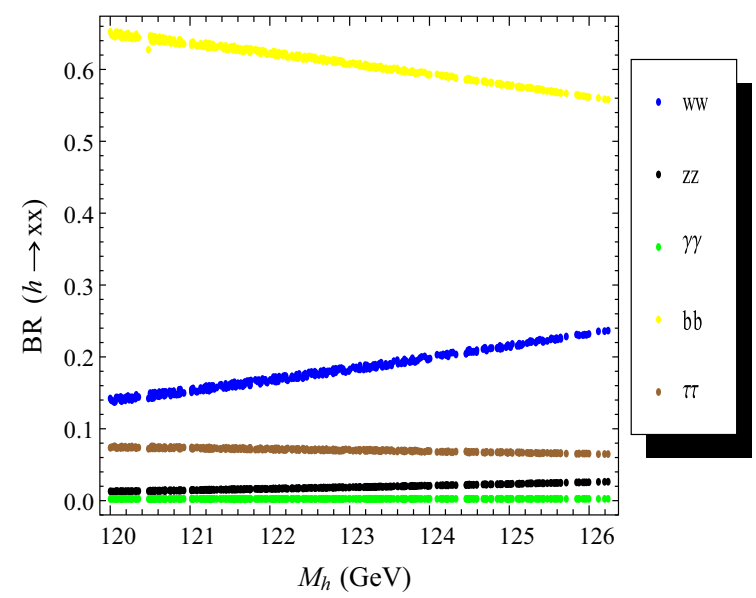

Fig. 5 Left: The dominant branching ratios for our Higgs-like boson as function of its mass. Right: Decay signal strengths taking into account the combination of ATLAS and CMS data. The error bars indicate the

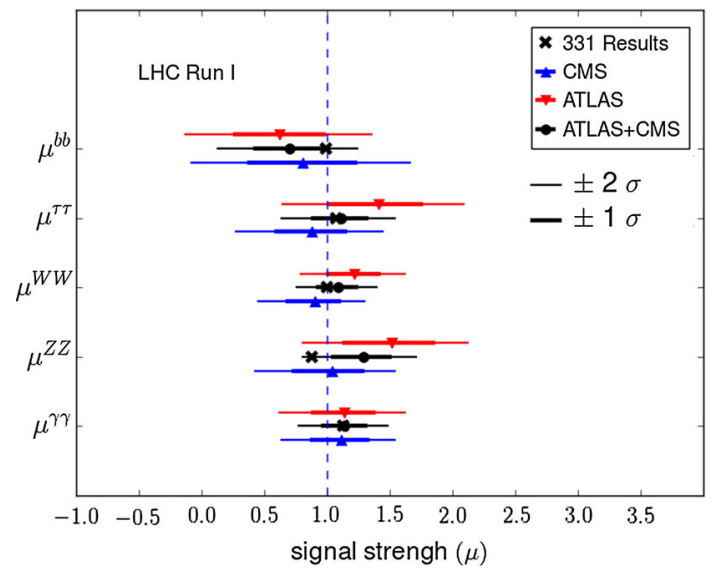

$1 \sigma$ (thick lines) and $2 \sigma$ (thin lines) intervals. The combined results show a remarkable agreement with the SM prediction (normalized to $\mu=1$ )

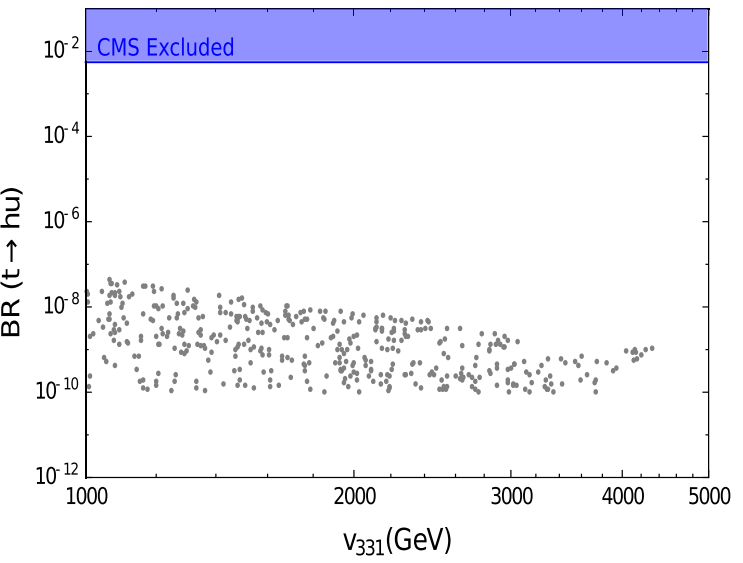

Fig. 6 Calculation of the branching ratios for the top decays $t \rightarrow h c$ and $t \rightarrow h u$

ing quarks already at tree level. This is a consequence of quark masses having more than one source, as depicted in the superpotential of the model. According to the last line of the superpotential, and the fact that our Higgs-like boson is mostly a composition of $R_{\eta^{\prime}}$ and $R_{\rho^{\prime}}$, the dominant flavor changing processes are those involving the third family of quarks. We studied all the possible processes here and obtained that the decays $h \rightarrow b s, b d$ are very suppressed even in relation to the loop predictions of the SM [30]. Thus, the significant processes are those involving the Yukawa interactions $t-h-u$ and $t-h-c$. For this case, the signature of our Higgs-like boson is through top quark decays via Higgs-mediated flavor-changing processes [31]. The behavior of the branching ratio of these decays as function of $v_{331}$ is presented in Fig. 6.

Although our results are far below the excluded region, we hope this to be probed in the next generation of collider.

\section{Stability of the potential}

In this section we check if that region of parameter space considered throughout this paper is so that the potential of the model is stable for such value of the parameters. In practical terms, we have to be sure that the minimum of the potential we are considering is in fact the global one. We check this by means of the Vevacious 1.2.02 Package [32,33]. We export the model implemented in the Sarah package to the Vevacious one and scanned that region of the parameter space used in the results above. Our result is presented in the plane $\tan (\beta)$ versus $v_{331}$ displayed in Fig. 7 .

Perceive that, except for a small region of the parameter space presenting long lived behavior, but with decay time larger than the age of our Universe (blue region), major part of the region of the parameter space considered in this work is associated to a stable minimum of the potential. 


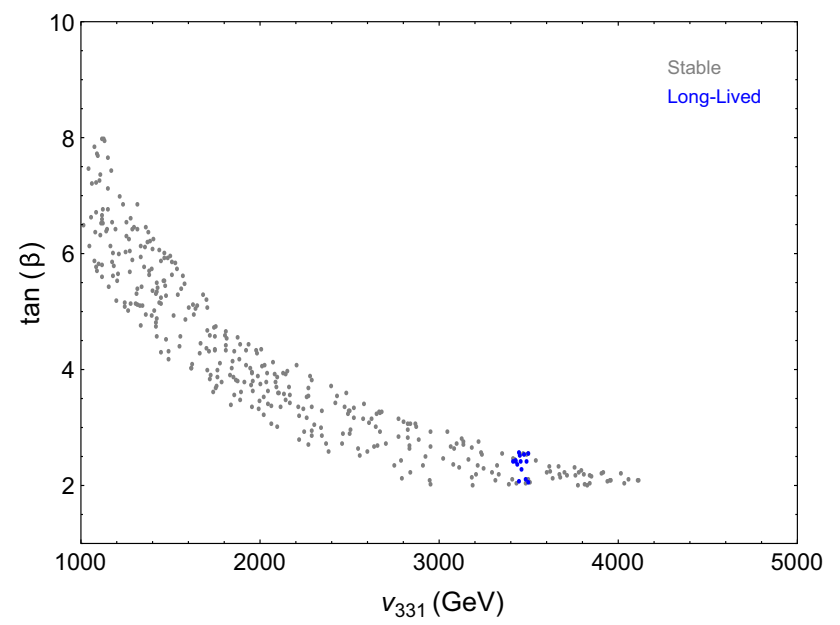

Fig. 7 Points in the plane $\tan (\beta)$ versus $v_{331}$ that guarantee stability of the potential

\section{Conclusions}

In this work we developed the Higgs sector of the minimal SUSY331 model with the focus on the lightest Higgs. We obtained its mass and couplings with the standard particles. With regard to its mass, tree level contribution may surpass the usual MSSM prediction and attain more than $100 \mathrm{GeV}$. Consequently, a $125 \mathrm{GeV}$ Higgs will demand feeble loop corrections. In our calculations we showed that a $125 \mathrm{GeV}$ Higgs is compatible with stop with mass below the TeV scale running in the loops. This is a remarkable result concerning the naturalness principle.

Although Higgs mass is the most important aspect of the Higgs physics, a complete work demands we extend our investigation to the Higgs couplings with the standard particles. We performed such an analysis and confirmed that they recover the observed pattern of branching ratios and signal strengths for its dominant processes. Finally we discussed the signature of our Higgs-like boson which manifests in the form of flavor changing processes intermediated by the Higgs. The most relevant ones are the top decay channels $t \rightarrow h+u$ and $t \rightarrow h+c$. We studied the behavior of these processes and concluded that they are out of reach of the LHC but, perhaps, may be probed in future colliders. We also checked if the region of parameter space considered in this work is compatible with the stability of the potential. We obtained that, except for a small region of the parameter space presenting long lived behavior, but with decay time larger than the age of our Universe, major part of it belongs to those points where the potential has a stable global minimum. All of this leads us to conclude that the minimal SUSY331 model is an interesting supersymmetric model for particle physics.
Acknowledgements We thank Felipe Ferreira and Antonio Santos for useful discussions. This work was supported by Conselho Nacional de Pesquisa e Desenvolvimento Científico- CNPq (C.A.S.P, P.S.R.S.). CS is supported by CAPES/PDSE Process 88881.134759/2016-01.

Open Access This article is distributed under the terms of the Creative Commons Attribution 4.0 International License (http://creativecomm ons.org/licenses/by/4.0/), which permits unrestricted use, distribution, and reproduction in any medium, provided you give appropriate credit to the original author(s) and the source, provide a link to the Creative Commons license, and indicate if changes were made.

Funded by SCOAP ${ }^{3}$.

\section{Appendix A: Tadpole equations}

$$
\begin{aligned}
& \left\langle\frac{\partial V}{\partial \rho^{0}}\right\rangle_{0}=\left(m_{\rho}^{2}+\mu_{\rho}^{2}\right) v_{\rho}+b_{\rho} v_{\rho^{\prime}} \\
& +\frac{1}{12} g^{2} v_{\rho}\left(-v_{\eta}^{2}+v_{\eta^{\prime}}^{2}-v_{\chi}^{2}+v_{\chi^{\prime}}^{2}+2 v_{\rho}^{2}-2 v_{\rho^{\prime}}^{2}\right) \\
& +\frac{1}{2} g_{N}^{2} v_{\rho}\left(-v_{\chi}^{2}+v_{\chi^{\prime}}^{2}+v_{\rho}^{2}-v_{\rho^{\prime}}^{2}\right) \\
& +\frac{1}{\sqrt{2}} f_{1}^{\prime}\left(v_{\eta^{\prime}} v_{\chi^{\prime}} \mu_{\rho}\right)+\frac{1}{\sqrt{2}} f_{1}\left(\frac{1}{\sqrt{2}} v_{\eta}^{2} v_{\rho}\right. \\
& \left.+\frac{1}{\sqrt{2}} v_{\chi}^{2} v_{\rho}+v_{\eta} v_{\chi^{\prime}} \mu_{\chi}+v_{\eta^{\prime}} v_{\chi} \mu_{\eta}\right) \\
& +\frac{1}{\sqrt{2}} k_{1} v_{\eta} v_{\chi}=0 \text {, } \\
& \left\langle\frac{\partial V}{\partial \rho^{\prime 0}}\right\rangle_{0}=\left(m_{\rho^{\prime}}^{2}+\mu_{\rho}^{2}\right) v_{\rho^{\prime}}+b_{\rho} v_{\rho} \\
& +\frac{1}{12} g^{2} v_{\rho^{\prime}}\left(v_{\eta}^{2}-v_{\eta^{\prime}}^{2}+v_{\chi}^{2}-v_{\chi^{\prime}}^{2}-2 v_{\rho}^{2}+2 v_{\rho^{\prime}}^{2}\right) \\
& +\frac{1}{2} g_{N}^{2} v_{\rho^{\prime}}\left(-v_{\chi}^{2}+v_{\chi^{\prime}}^{2}+v_{\rho}^{2}-v_{\rho^{\prime}}^{2}\right) \\
& +\frac{1}{\sqrt{2}} f_{1}\left(v_{\eta} v_{\chi} \mu_{\rho}\right)+\frac{1}{\sqrt{2}} f_{1}^{\prime}\left(\frac{1}{\sqrt{2}} v_{\eta^{\prime}}^{2} v_{\rho^{\prime}}+\frac{1}{\sqrt{2}} v_{\chi^{\prime}}^{2} v_{\rho^{\prime}}\right. \\
& \left.+v_{\eta} v_{\chi^{\prime}} \mu_{\eta}+v_{\eta^{\prime}} v_{\chi} \mu_{\chi}\right) \\
& +\frac{1}{\sqrt{2}} k_{2} v_{\eta^{\prime}} v_{\chi^{\prime}}=0 \\
& \left\langle\frac{\partial V}{\partial \eta^{0}}\right\rangle_{0}=\left(m_{\eta}^{2}+\mu_{\eta}^{2}\right) v_{\eta}+b_{\eta} v_{\eta^{\prime}} \\
& +\frac{1}{12} g^{2} v_{\eta}\left(-v_{\rho}^{2}+v_{\rho^{\prime}}^{2}-v_{\chi}^{2}+v_{\chi^{\prime}}^{2}+2 v_{\eta}^{2}-2 v_{\eta^{\prime}}^{2}\right) \\
& +\frac{1}{2} f_{1}^{\prime}\left(v_{\rho^{\prime}} v_{\chi^{\prime}} \mu_{\eta}\right)+\frac{1}{\sqrt{2}} f_{1}\left(\frac{1}{\sqrt{2}} v_{\rho}^{2} v_{\eta}+\frac{1}{\sqrt{2}} v_{\chi}^{2} v_{\eta}\right. \\
& \left.+v_{\rho} v_{\chi^{\prime}} \mu_{\chi}+v_{\rho^{\prime}} v_{\chi} \mu_{\rho}\right)+\frac{1}{\sqrt{2}} k_{1} v_{\rho} v_{\chi}=0, \\
& \left\langle\frac{\partial V}{\partial \eta^{\prime 0}}\right\rangle_{0}=\left(m_{\eta^{\prime}}^{2}+\mu_{\eta}^{2}\right) v_{\eta^{\prime}}+b_{\eta} v_{\eta}+\frac{1}{12} g^{2} v_{\eta^{\prime}} \\
& \left(v_{\rho}^{2}-v_{\rho^{\prime}}^{2}+v_{\chi}^{2}-v_{\chi^{\prime}}^{2}-2 v_{\eta}^{2}+2 v_{\eta^{\prime}}^{2}\right) \\
& +\frac{1}{\sqrt{2}} f_{1}\left(v_{\rho} v_{\chi} \mu_{\eta}\right)+\frac{1}{\sqrt{2}} f_{1}^{\prime}\left(\frac{1}{\sqrt{2}} v_{\rho^{\prime}}^{2} v_{\eta^{\prime}}+\frac{1}{\sqrt{2}} v_{\chi^{\prime}}^{2} v_{\eta^{\prime}}\right. \\
& \left.+v_{\rho} v_{\chi^{\prime}} \mu_{\rho}+v_{\rho^{\prime}} v_{\chi} \mu_{\chi}\right)+\frac{1}{\sqrt{2}} k_{2} v_{\rho^{\prime}} v_{\chi^{\prime}}=0, \\
& \left\langle\frac{\partial V}{\partial \chi^{0}}\right\rangle_{0}=\left(m_{\chi}^{2}+\mu_{\chi}^{2}\right) v_{\chi}+b_{\chi} v_{\chi^{\prime}}+\frac{1}{12} g^{2} v_{\chi}\left(-v_{\eta}^{2}+v_{\eta^{\prime}}^{2}-v_{\rho}^{2}\right. \\
& \left.+v_{\rho^{\prime}}^{2}+2 v_{\chi}^{2}-2 v_{\chi^{\prime}}^{2}\right)+\frac{1}{2} g_{N}^{2} v_{\chi}\left(v_{\chi}^{2}-v_{\chi^{\prime}}^{2}-v_{\rho}^{2}+v_{\rho^{\prime}}^{2}\right)
\end{aligned}
$$




$$
\begin{aligned}
+ & \frac{1}{\sqrt{2}} f_{1}^{\prime}\left(v_{\eta^{\prime}} v_{\rho^{\prime}} \mu_{\chi}\right)+\frac{1}{\sqrt{2}} f_{1}\left(\frac{1}{\sqrt{2}} v_{\eta}^{2} v_{\chi}\right. \\
& \left.+\frac{1}{\sqrt{2}} v_{\rho}^{2} v_{\chi}+v_{\eta} v_{\rho^{\prime}} \mu_{\rho}+v_{\eta^{\prime}} v_{\rho} \mu_{\eta}\right) \\
& +\frac{1}{\sqrt{2}} k_{1} v_{\eta} v_{\rho}=0, \\
\left\langle\frac{\partial V}{\partial \chi^{\prime 0}}\right\rangle_{0}= & \left(m_{\chi^{\prime}}^{2}+\mu_{\chi^{\prime}}^{2}\right) v_{\chi^{\prime}}+b_{\chi} v_{\chi} \\
& +\frac{1}{12} g^{2} v_{\chi^{\prime}}\left(v_{\eta}^{2}-v_{\eta^{\prime}}^{2}+v_{\rho}^{2}-v_{\rho^{\prime}}^{2}-2 v_{\chi}^{2}+2 v_{\chi^{\prime}}^{2}\right)
\end{aligned}
$$

$$
\begin{aligned}
& +\frac{1}{2} g_{N}^{2} v_{\chi^{\prime}}\left(-v_{\chi}^{2}+v_{\chi^{\prime}}^{2}+v_{\rho}^{2}-v_{\rho^{\prime}}^{2}\right) \\
& +\frac{1}{\sqrt{2}} f_{1}\left(v_{\eta} v_{\rho} \mu_{\chi}\right) \\
& +\frac{1}{\sqrt{2}} f_{1}^{\prime}\left(\frac{1}{\sqrt{2}} v_{\eta^{\prime}}^{2} v_{\chi^{\prime}}+\frac{1}{\sqrt{2}} v_{\rho^{\prime}}^{2} v_{\chi^{\prime}}\right. \\
& \left.+v_{\eta} v_{\rho^{\prime}} \mu_{\eta}+v_{\eta^{\prime}} v_{\rho} \mu_{\rho}\right)+\frac{1}{\sqrt{2}} k_{2} v_{\eta^{\prime}} v_{\rho^{\prime}}=0 .
\end{aligned}
$$

\section{Appendix B: SARAH model file}

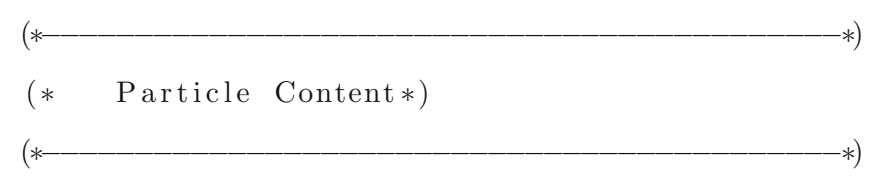

(* Global symmetries *)

Global $[[1]]=\{\mathrm{Z}[2]$, RParity $\}$

$\mathrm{RpM}=\{-1,-1,1\} ;$

$\mathrm{RpP}=\{1,1,-1\}$;

(* Gauge Superfields *)

Gauge $[[1]]=\{\mathrm{G}, \mathrm{SU}[3], \quad$ color, g3, False, $\mathrm{RpM}\}$;

Gauge $[[2]]=\{\mathrm{WB}, \quad \mathrm{SU}[3], \quad$ left $, \quad \mathrm{g} 2, \quad$ True, $\mathrm{RpM}\}$;

Gauge $[[3]]=\{\mathrm{B}, \quad \mathrm{U}[1]$, xcharge, $\mathrm{g} 1$, False, $\mathrm{RpM}\}$;

(* Chiral Superfields *)

(* Fermions *)

SuperFields $[[1]]=\{\mathrm{q} 1, \quad 1,\{\mathrm{jj} 1 \mathrm{~L}, \quad-\mathrm{u} 1 \mathrm{~L}, \mathrm{~d} 1 \mathrm{~L}\}, 3,-3,-1 / 3, \mathrm{RpM}\}$;

SuperFields $[[2]]=\{\mathrm{q} 2, \quad 1,\{\mathrm{jj} 2 \mathrm{~L}, \quad-\mathrm{u} 2 \mathrm{~L}, \mathrm{~d} 2 \mathrm{~L}\}, 3,-3,-1 / 3, \mathrm{RpM}\}$;

SuperFields $[[3]]=\{\mathrm{q} 3, \quad 1,\{\mathrm{jj} 3 \mathrm{~L}, \quad \mathrm{~d} 3 \mathrm{~L}, \mathrm{u} 3 \mathrm{~L}\}, 3,3,2 / 3, \mathrm{RpM}\}$;

SuperFields $[[4]]=\{\operatorname{lep}, 3,\{\operatorname{conj}[\mathrm{eR}], \quad \mathrm{eL}, \mathrm{vL}\}, 1,3, \quad 0, \mathrm{RpM}\}$;

SuperFields $[[5]]=\{\mathrm{dr}, 3, \operatorname{conj}[\mathrm{dR}], \quad-3,1,1 / 3, \mathrm{RpM}\}$;

SuperFields $[[8]]=\{\operatorname{ur}, 3, \operatorname{conj}[u R], \quad-3,1,-2 / 3, \operatorname{RpM}\}$;

SuperFields $[[11]]=\{\mathrm{jjr}, 2, \operatorname{conj}[\mathrm{jjR}], \quad-3,1,4 / 3, \mathrm{RpM}\}$;

SuperFields $[[13]]=\{\mathrm{jj} 3,1, \operatorname{conj}[\mathrm{jj} 3 \mathrm{R}],-3,1,-5 / 3, \mathrm{RpM}\}$;

(* Scalar *)

SuperFields $[[14]]=\{$ Rho, $1,\{$ Rhopp,$\quad$ Rho0, $\operatorname{Rhop}\}, \quad 1, \quad 3, \quad 1, \operatorname{RpP}\}$;

SuperFields $[[15]]=\{$ Chi $, \quad 1,\{$ Chi0, Chimm, $\operatorname{Chim}\}, \quad 1, \quad 3,-1, \operatorname{RpP}\} ;$

SuperFields $[[16]]=\{$ Rho1, 1, Rholmm, Rho10, Rho1m $\}, 1,-3,-1, \operatorname{RpP}\}$;

SuperFields $[[17]]=\{$ Chi1, 1, $\{$ Chi10, Chi1pp, Chi1p $\}, 1,-3, \quad 1, \operatorname{RpP}\}$;

SuperFields $[[18]]=\{$ Eta $, 1,\{$ eta $2 \mathrm{p}$, eta $1 \mathrm{~m}$, eta 0$\}, 1,3, \quad 0, \operatorname{RpP}\}$;

SuperFields $[[19]]=\{$ Eta1, $1,\{$ eta2m, eta1p, eta10 $\}, 1,-3,0, \operatorname{RpP}\} ;$ 


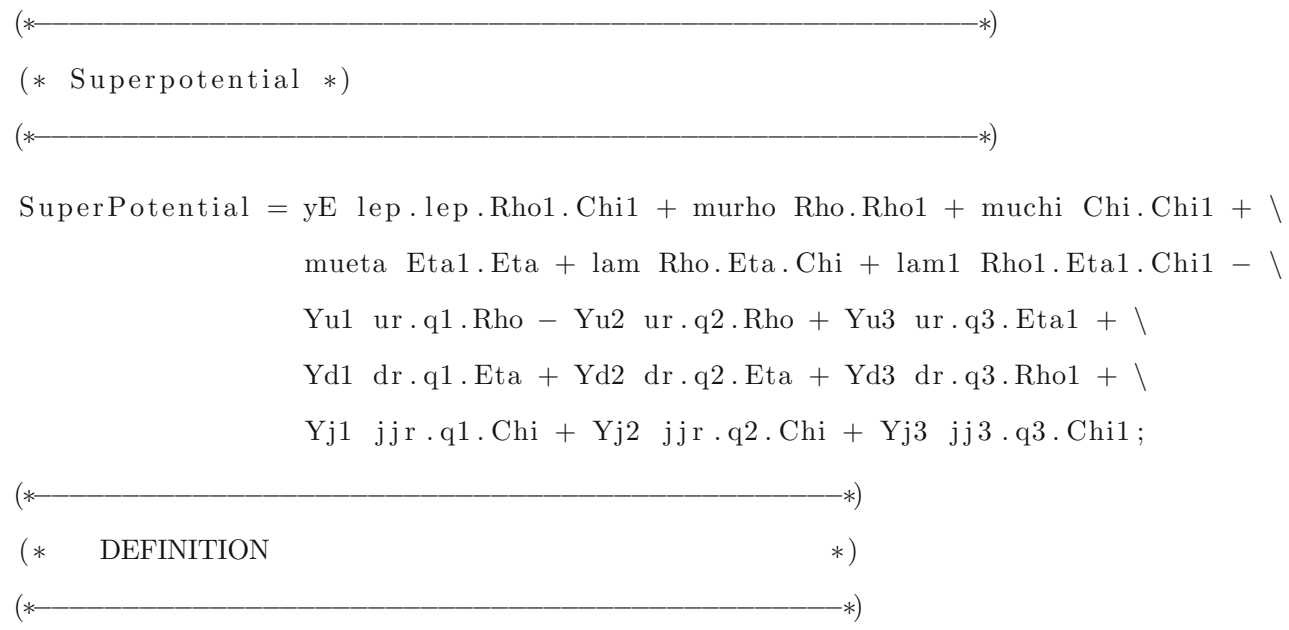

NameOfStates $=\{$ GaugeES, EWSB $\}$;

(* - - Before EWSB —— $*$ )

DEFINITION [GaugeES ] [ DiracSpinors $]=\{$

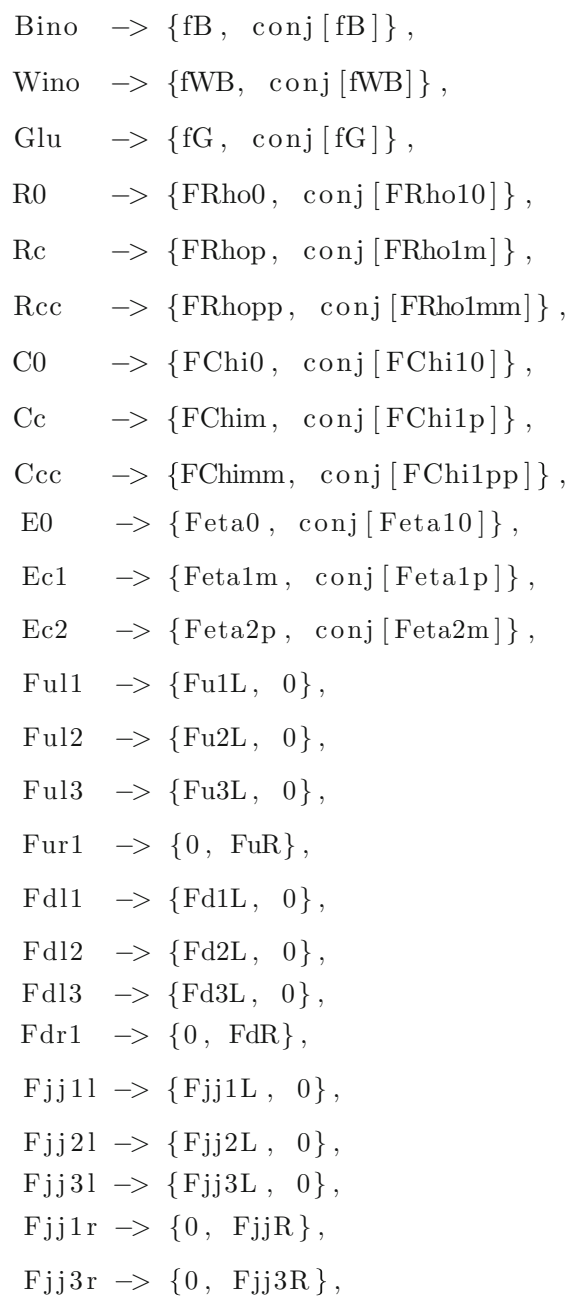




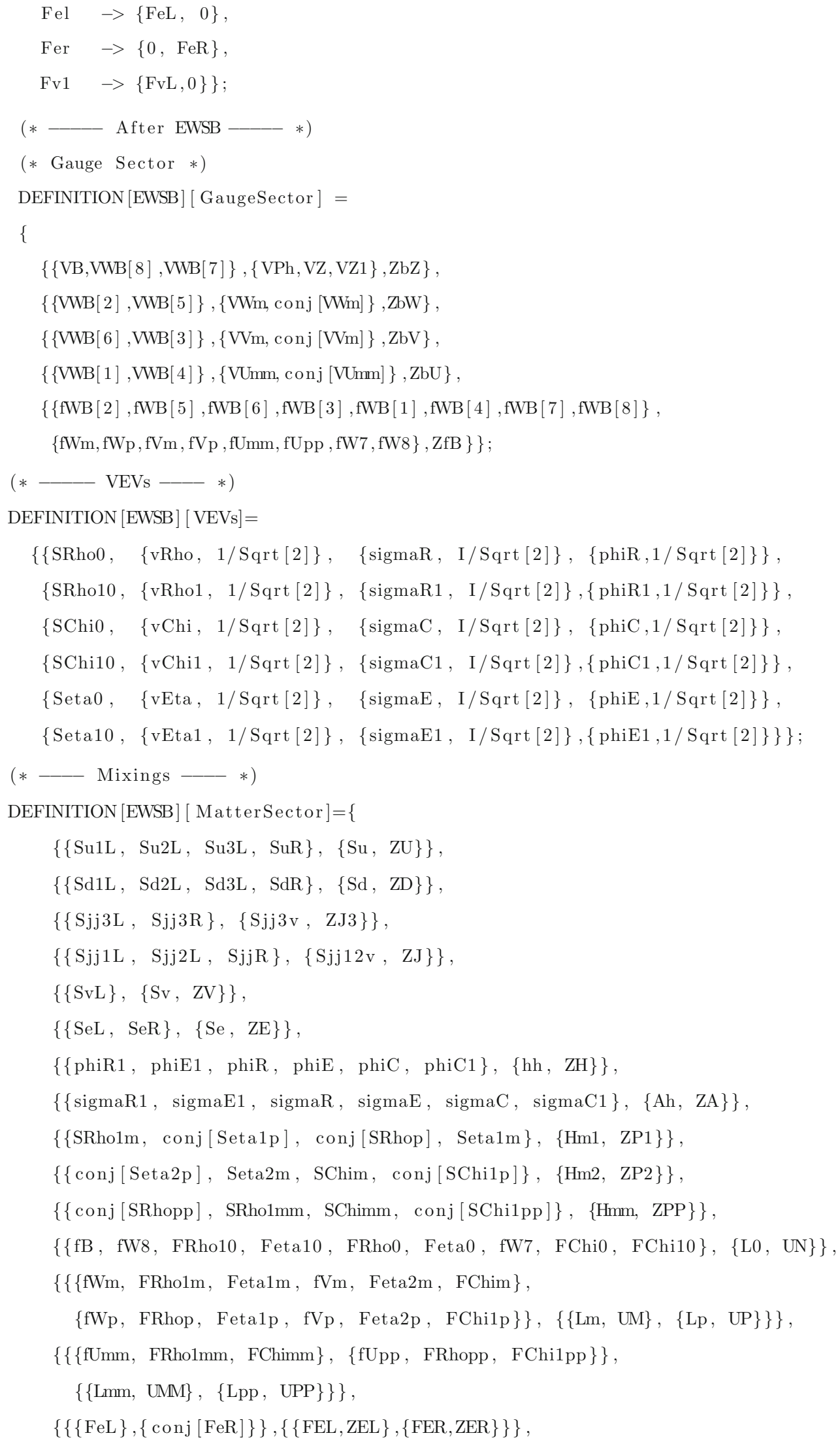


$\{\{\{$ Fjj1L, Fjj2L $\},\{\operatorname{conj}[F j j R]\}\},\{\{F J L, Z J L\},\{F J R, Z J R\}\}\}$,

$\{\{\{\mathrm{Fd} 1 \mathrm{~L}, \mathrm{Fd} 2 \mathrm{~L}, \mathrm{Fd} 3 \mathrm{~L}\},\{\operatorname{conj}[\mathrm{FdR}]\}\},\{\{\mathrm{FDL}, \mathrm{ZDL}\},\{\mathrm{FDR}, \mathrm{ZDR}\}\}\}$,

$\{\{\{\mathrm{Fu} 1 \mathrm{~L}$, Fu2L, Fu3L $\},\{\operatorname{conj}[\mathrm{FuR}]\}\},\{\{\mathrm{FUL}, \mathrm{ZUL}\},\{$ FUR, ZUR $\}\}\}\} ;$

DEFINITION $[$ EWSB $][$ Phases $]=\{$

$\{$ fG, PhaseGlu $\}$;

DEFINITION [EWSB] [ DiracSpinors $]=\{$

$\mathrm{Fd} \quad \rightarrow\{$ FDL, $\operatorname{conj}[$ FDR $]\}$,

$\mathrm{Fe} \rightarrow\{$ FEL, $\operatorname{conj}[\mathrm{FER}]\}$,

$\mathrm{Fu} \rightarrow\{$ FUL, $\operatorname{conj}[\mathrm{FUR}]\}$,

Fjj12 $\rightarrow\{$ FJL, conj $[$ FJR $]\}$,

Fjj3 $\rightarrow\{F j j 3 L, F j j 3 R\}$,

$\mathrm{Fv} \rightarrow\{\mathrm{FvL}, 0\}$,

Neu $\rightarrow\{$ Lo, $\operatorname{conj}[$ Lo $]\}$,

Cha $\rightarrow\{\operatorname{Lm}, \quad \operatorname{conj}[\mathrm{Lp}]\}$,

DCha $\rightarrow\{$ Lmm, $\operatorname{conj}[$ Lpp $]\}$,

Glu $\rightarrow\{$ fG, $\operatorname{conj}[\mathrm{fG}]\}\}$; 


\section{Appendix C: SPheno input for SARAH}

OnlyLowEnergySPheno = True;

$\mathrm{MINPAR}=\{$

$\{1, \quad \mathrm{v} 331 \mathrm{IN}\}$,

$\{2$, TanBeta $\}$,

$\{3$, TanBetaR $\}$,

$\{4$, TanBetaE $\}$,

$\{5$, TanBetaC $\}$,

$\{10$, Azero $\}$,

$\{11$, mSUSYL $\}$,

$\{12, \operatorname{mSUSYR}\}$,

$\{21$, M1input $\}$,

$\{22$, M2input $\}$,

$\{23$, M3input $\}$,

$\{31$, MJ1in $\}$,

$\{32$, MJ2in $\}$,

$\{33$, MJ3in $\}$,

\{41, muRinput $\}$,

$\{42$, muEinput $\}$,

$\{43$, muCinput $\}$,

$\{44$, Bzero $\}$,

$\{45$, finput $\}$,

$\{46$, fpinput $\}$,

$\{47$, Tfinput $\}$,

$\{48$, Tfpinput $\}$,

$\{51, \mathrm{Xt}\}\}$;

RealParameters $=\{$ v331IN, TanBeta, TanBetaR, TanBetaE, TanBetaC $\}$;

DEFINITION [ MatchingConditions $]=\{$

$\left\{\mathrm{g} 1, \mathrm{~g} 1 \mathrm{SM} * \mathrm{~g} 2 \mathrm{SM} / \mathrm{Sqrt}\left[\mathrm{g}^{\mathrm{SSM}}{ }^{\wedge} 2-3 * \mathrm{~g} 1 \mathrm{SM} \wedge 2\right]\right\}$,

$\{\mathrm{g} 2, \mathrm{~g} 2 \mathrm{SM}\}$,

$\{\mathrm{g} 3, \mathrm{~g} 3 \mathrm{SM}\}$,

$\{$ vRho, $\operatorname{vSM} * \operatorname{Cos}[\operatorname{ArcTan}[\operatorname{TanBeta}]] * \operatorname{Cos}[\operatorname{ArcTan}[\operatorname{TanBetaR}]]\}$,

$\{$ vRho1, vSM*Cos [ArcTan [TanBeta $]] * \operatorname{Sin}[\operatorname{ArcTan}[$ TanBetaR $]]\}$,

$\{$ vEta, vSM*Sin $[\operatorname{ArcTan}[$ TanBeta $]] * \operatorname{Cos}[\operatorname{ArcTan}[$ TanBetaE $]]\}$,

$\{$ vEta1, vSM $* \operatorname{Sin}[\operatorname{ArcTan}[$ TanBeta $]] * \operatorname{Sin}[\operatorname{ArcTan}[$ TanBetaE $]]\}$, 
$\{\mathrm{yE}, \operatorname{Sqrt}[2] * \mathrm{YeSM} * \mathrm{vSM} /(\mathrm{vRho} 1 * \mathrm{vChi1})\}$,

$\{\mathrm{Yu} 1[1], \mathrm{YuSM}[1,1] * \mathrm{vSM} / \mathrm{vRho}\}$,

$\{\mathrm{Yu} 1[2], \mathrm{YuSM}[2,1] * \mathrm{vSM} / \mathrm{vRho}\}$,

$\{\mathrm{Yu} 1[3], \operatorname{YuSM}[3,1] * \mathrm{vSM} / \mathrm{vRho}\}$,

$\{\mathrm{Yu} 2[1], \mathrm{YuSM}[1,2] * \mathrm{vSM} / \mathrm{vRho}\}$,

$\{\mathrm{Yu} 2[2], \operatorname{YuSM}[2,2] * \mathrm{vSM} / \mathrm{vRho}\}$,

$\{\mathrm{Yu} 2[3], \operatorname{YuSM}[3,2] * \mathrm{vSM} / \mathrm{vRho}\}$,

$\{\mathrm{Yu} 3[1], \mathrm{YuSM}[1,3] * \mathrm{vSM} / \mathrm{vEta} 1\}$,

$\{\mathrm{Yu} 3[2], \operatorname{YuSM}[2,3] * \mathrm{vSM} / \mathrm{vEta} 1\}$,

$\{\mathrm{Yu} 3[3], \operatorname{YuSM}[3,3] * \mathrm{vSM} / \mathrm{vEta} 1\}$,

$\{\operatorname{Yd} 1[1], \operatorname{YdSM}[1,1] * \mathrm{vSM} / \mathrm{vEta}\}$,

$\{\mathrm{Yd} 1[2], \operatorname{YdSM}[2,1] * \mathrm{vSM} / \mathrm{vEta}\}$,

$\{\mathrm{Yd} 1[3], \operatorname{YdSM}[3,1] * \mathrm{vSM} / \mathrm{vEta}\}$,

$\{\mathrm{Yd} 2[1], \operatorname{YdSM}[1,2] * \mathrm{vSM} / \mathrm{vEta}\}$,

$\{\mathrm{Yd} 2[2], \operatorname{YdSM}[2,2] * \mathrm{vSM} / \mathrm{vEta}\}$,

$\{\mathrm{Yd} 2[3], \operatorname{YdSM}[3,2] * \mathrm{vSM} / \mathrm{vEta}\}$,

$\{\mathrm{Yd} 3[1], \operatorname{YdSM}[1,3] * \mathrm{vSM} / \mathrm{vRho1}\}$,

$\{\mathrm{Yd} 3[2], \operatorname{YdSM}[2,3] * \mathrm{vSM} / \mathrm{vRho1}\}$,

$\{\mathrm{Yd} 3[3], \operatorname{YdSM}[3,3] * \mathrm{vSM} / \mathrm{vRho1}\}\}$;

BoundaryLowScaleInput $=\{$

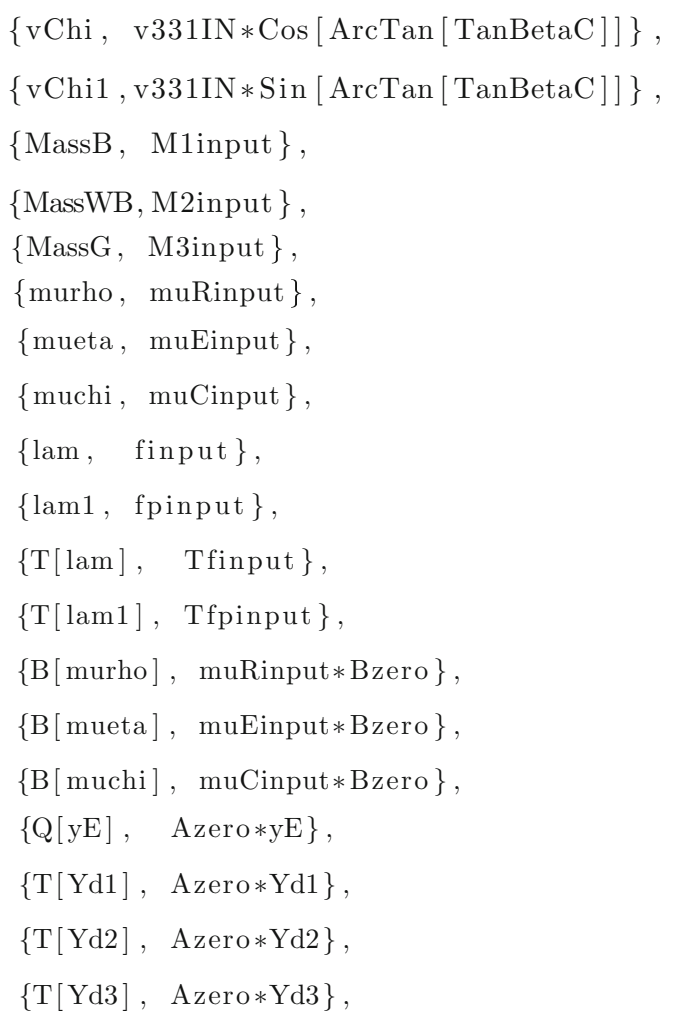




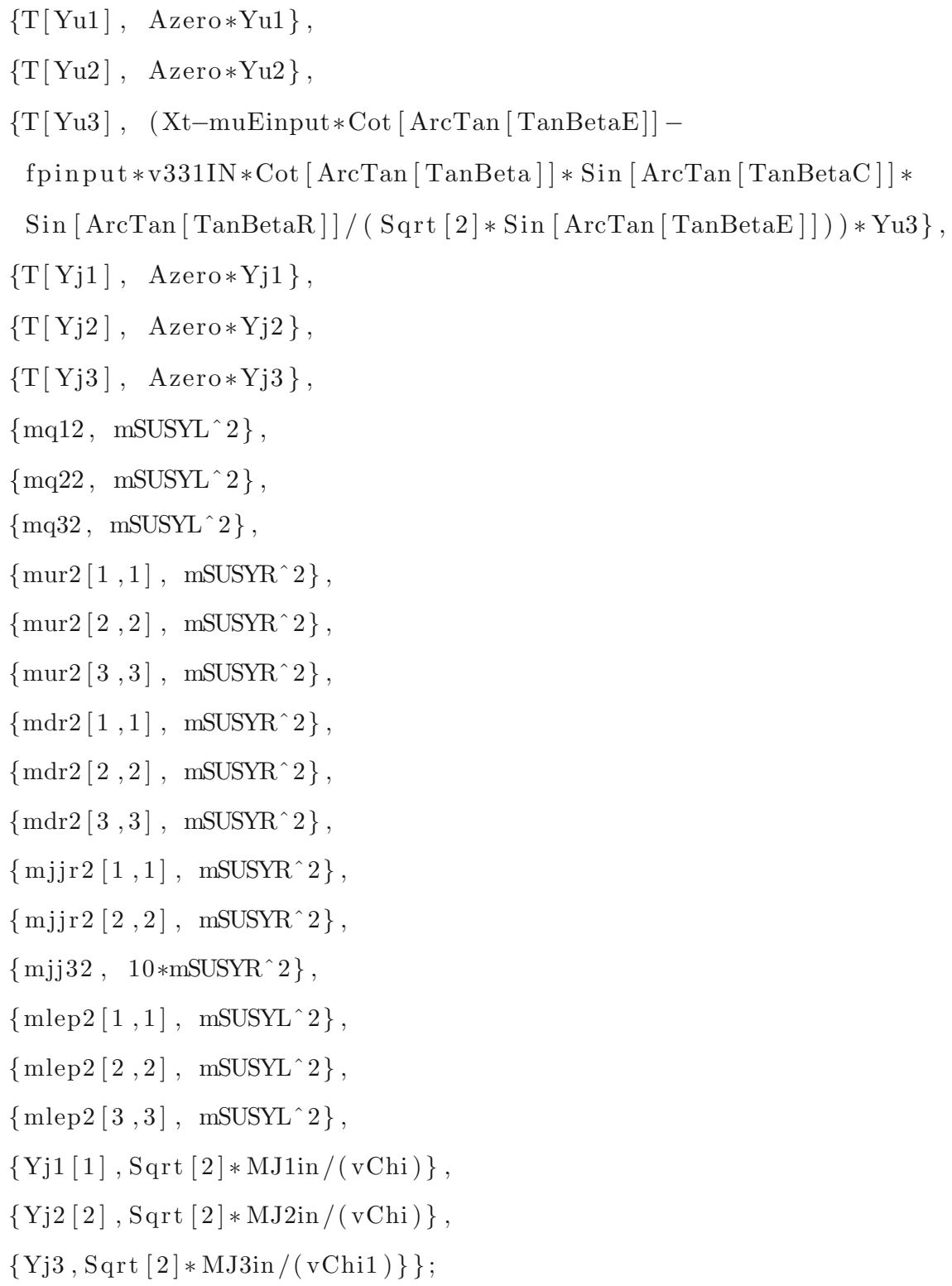




\section{References}

1. G. Aad et al. (ATLAS), Phys. Lett. B 716, 1 (2012). arXiv: 1207.7214

2. S. Chatrchyan et al. (CMS), Phys. Lett. B 716, 30 (2012). arXiv: 1207.7235

3. G. Aad et al. (ATLAS, CMS), JHEP 08, 045 (2016a). arXiv: 1606.02266

4. M. Carena, H.E. Haber, Prog. Part. Nucl. Phys. 50, 63 (2003). arXiv:hep-ph/0208209

5. F. Pisano, V. Pleitez, Phys. Rev. D 46, 410 (1992). arXiv:hep-ph/9206242

6. P.H. Frampton, Phys. Rev. Lett. 69, 2889 (1992)

7. C.A. de Sousa Pires, O.P. Ravinez, Phys. Rev. D 58, 035008 (1998)

8. C.A. de Sousa Pires, O.P. Ravinez, Phys. Rev. D 58, 35008 (1998). arXiv:hep-ph/9803409

9. C.A. de Sousa, Phys. Rev. D 60, 075013 (1999). arXiv:hep-ph/9902406

10. P.B. Pal, Phys. Rev. D 52, 1659 (1995). arXiv:hep-ph/9411406

11. A.G. Dias, V. Pleitez, Phys. Rev. D 69, 077702 (2004). arXiv:hep-ph/0308037

12. A.G. Dias, C.A.de S. Pires, P.S. Rodrigues da Silva, Phys. Rev. D 68, 115009 (2003). arXiv:hep-ph/0309058

13. J.G. Ferreira, P.S. Rodrigues da Silva, C.A. de S. Pires, J.G. Rodrigues, Phys. Lett. B 771, 199 (2017). arXiv:1612.01463

14. T.V. Duong, E. Ma, Phys. Lett. B 316, 307 (1993). arXiv:hep-ph/9306264

15. J.C. Montero, V. Pleitez, M.C. Rodriguez, Phys. Rev. D 65, 035006 (2002a). arXiv:hep-ph/0012178

16. D.T. Huong, L.T. Hue, M.C. Rodriguez, H.N. Long, Nucl. Phys. B 870, 293 (2013). arXiv: 1210.6776

17. J.G. Ferreira, C.A.de S. Pires, P.S. Rodrigues da Silva, A. Sampieri, Phys. Rev. D 88, 105013 (2013). arXiv:1308.0575
18. J.C. Montero, C.A.de S. Pires, V. Pleitez, Phys. Rev. D 66, 113003 (2002b). arXiv:hep-ph/0112203

19. F. Queiroz, C.A.de S. Pires, P.S.R. da Silva, Phys. Rev. D 82, 065018 (2010). arXiv:1003.1270

20. P.V. Dong, D.T. Si, Phys. Rev. D 90, 117703 (2014). arXiv: 1411.4400

21. F. Staub, (2008). arXiv:0806.0538

22. F. Staub, Adv. High Energy Phys. 2015, 840780 (2015). arXiv: 1503.04200

23. A. Vicente, (2015). arXiv: 1507.06349

24. W. Porod, Comput. Phys. Commun. 153, 275 (2003). arXiv:hep-ph/0301101

25. W. Porod, F. Staub, Comput. Phys. Commun. 183, 2458 (2012). arXiv: 1104.1573

26. F. Staub, T. Ohl, W. Porod, C. Speckner, Comput. Phys. Commun. 183, 2165 (2012). arXiv: 1109.5147

27. J.R. Andersen et al. (LHC Higgs Cross Section Working Group), (2013). arXiv:1307.1347

28. G. Aad et al., ATLAS. Eur. Phys. J. C 76, 6 (2016b). arXiv: 1507.04548

29. C. Collaboration (CMS) (2014)

30. L.G. Benitez-Guzmán, I. García-Jiménez, M.A. López-Osorio, E. Martínez-Pascual, J.J. Toscano, J. Phys. G42, 085002 (2015). arXiv: 1506.02718

31. V. Khachatryan et al., (CMS). JHEP 02, 079 (2017). arXiv: 1610.04857

32. J.E. Camargo-Molina, B. Garbrecht, B. O'Leary, W. Porod, F. Staub, Phys. Lett. B 737, 156 (2014). arXiv:1405.7376

33. J.E. Camargo-Molina, B. O'Leary, W. Porod, F. Staub, Eur. Phys. J. C 73, 2588 (2013). arXiv:1307.1477 